\title{
Not Going to Take This Anymore: Multi-objective Overtime Planning for Software Engineering Projects
}

\author{
Filomena Ferrucci*, Mark Harman ${ }^{\dagger}$, Jian Ren ${ }^{\dagger}$, and Federica Sarro* \\ ${ }^{*}$ University of Salerno, Fisciano (SA), Italy \\ $\dagger$ University College London, CREST centre, London, WC1E 6BT, UK
}

\begin{abstract}
Software Engineering and development is wellknown to suffer from unplanned overtime, which causes stress and illness in engineers and can lead to poor quality software with higher defects. In this paper, we introduce a multi-objective decision support approach to help balance project risks and duration against overtime, so that software engineers can better plan overtime. We evaluate our approach on 6 real world software projects, drawn from 3 organisations using 3 standard evaluation measures and 3 different approaches to risk assessment. Our results show that our approach was significantly better $(p<0.05)$ than standard multi-objective search in $76 \%$ of experiments (with high Cohen effect size in $85 \%$ of these) and was significantly better than currently used overtime planning strategies in $100 \%$ of experiments (with high effect size in all). We also show how our approach provides actionable overtime planning results and investigate the impact of the three different forms of risk assessment.
\end{abstract}

\section{INTRODUCTION}

Poor overtime planning is particularly pernicious in the software industry. Facing a combination of estimate inaccuracy and time-to-market pressure, software engineers are often coerced into high levels of unplanned overtime, leading to dissatisfaction, depression, and defects. Inability to plan and budget for overtime leads to hastily arranged, unplanned overtime and the familiar spectre of the 'death march project' [1].

This has a detrimental effect on the quality of the lives of the software engineers unfortunate enough to be involved and also of the quality of the software that they produce. As might be expected, the problems of unplanned overtime have been widely reported upon in the occupational health literature, which contains many systematic studies of its unfortunate side effects on professionals and the products and services they provide [2], [3], [4]

There is also evidence for the harmful effects of unplanned overtime specifically on software engineering professionals and the software they produce, though it is perhaps surprising how little this phenomenon has been systematically studied, given the widespread belief that it is so prevalent [1], [5] A controlled study of 377 software engineers found positive correlations $(p<0.05)$ between unplanned overtime and several widely-used stress and depression indicators [6]. There is also evidence that the deployment of overtime can lead to increased software defect counts [7].

Fortunately, there is also case study evidence that proper planning leads, not only to greater software engineer job satisfaction, but also to improved customer satisfaction in the resulting software products [8]. Looking to the wider (non-software-engineering specific) literature, we can also find evidence that planned overtime has few, if any, of the harmful side-effects that so-often accompany unplanned overtime [9]. This evidence all points to the need for research into decision support for software engineers to help them better plan for overtime, balancing the need for overtime against project overrun risks and budgetary constraints.
The software engineering literature contains many excellent examples of research on software engineering support techniques for a wide range of engineering tasks such as testing, design and maintenance. This work has contributed to the software-enabled development environments that many software engineers now take for granted [10]. However, sadly, there has been no research aimed at providing support to software engineers in their attempts to plan for overtime.

This paper addresses this problem. We introduce an approach to support software engineers in better planning for overtime, while managing risk. The problem is to find the right balance between the conflicting objectives of reducing project duration, overtime, and risk.

Complex multi-objective decision problems with competing and conflicting constraints such as this are well suited to Search Based Software Engineering (SBSE) [11], which has proved able to provide decision support for other early-stage development activities, notably requirements engineering [12], [13], [14]. We believe that this is the first time that an approach has been introduced to provide decision support for software engineers attempting to reconcile these complex and difficult problems.

More specifically, the primary contributions of the paper are as follows:

1) We introduce a multi-objective search based formulation of the project overtime planning problem. Our approach is able to balance trade offs between project duration, overrun risk, and overtime resources for three different risk assessment models. It is applicable to standard software project plans, such as those constructed using the Critical Path Method, widely adopted by software engineers and implemented in many tools.

2) We present an empirical study on 6 real world software projects, ranging in size from a few person weeks to roughly four person years. This leads to 54 different experiments, comparing our proposed algorithm (with domain specific crossover operator) to the standard multiobjective algorithm and random search. The results reveal that our approach was significantly better than standard multi-objective search in $76 \%$ of experiments and was significantly better than random search in $100 \%$ experiments. The standard multi-objective approach significantly outperforms our approach in none of the experiments. We repeated the experiments to compare our approach with standard overtime planning strategies reported in the literature. This revealed that our approach always significantly outperforms these standard strategies with high effect size.

3) We present case studies using Pareto fronts obtained by our approach to illustrate how they yield actionable insights into project planning tradeoffs. We also use our approach to investigate the different risk assessment models that might be adopted. 
The rest of the paper is organised as follows: In Section II the overtime planning problem is defined. Section III introduces our search based approach to solving this problem using a multi-objective Pareto optimal approach. Section IV describes the method used in our empirical studies, the results of which are presented in Section V. Section VI analyses the limitations of the present study, while Section VII describes the context of related work in which the current paper is located. Section VIII concludes and presents directions for future work.

\section{Problem Formulation}

Our formulation of the problem starts from the Work Breakdown Schedule (WBS) produced by the software engineer, which we formalise here for clarity. Such a WBS can be produced by many project planning tools, such as Microsoft project (the tool used by all the organisations that provided the real world schedules used to evaluate our approach in this paper).

Let a project schedule be represented as an acyclic directed graph consisting of a node set $W P=\left\{w p_{1}, w p_{2}, \ldots, w p_{n}\right\}$ of work packages and an edge set $D E P=\left\{\left(w p_{i}, w p_{j}\right): i \neq j, 1 \leq i \leq n, 1 \leq j \leq n\right\}$ of dependencies between elements of WP, where $w p_{j}$ can start only when $w p_{i}$ has completed. WP and DEPS form a graph, the set of paths, $\Pi$, of which, denote the dependence-respecting orderings of work packages to be undertaken to complete the project. Associated with each work package, $w p_{i}$, is the estimated effort, $e_{i}$, required to complete $w p_{i}$ and also its estimated duration Duration $\left(w p_{i}\right)$. Based on this, the duration of each path $p \in \Pi$ through the project dependence graph is given by

$$
\text { Duration }_{p}=\sum_{\forall w p \in p} \operatorname{Duration}(w p)
$$

and the total estimated shortest possible duration of the project is given by any maximal length (or 'critical') path in $\Pi$. This is a formalisation of the well-known 'Critical Path Method' [15], which has been widely used in project planning for several decades. Though there may be several equal length critical paths (for which no other path is longer) it is traditional to select one and to refer to it as the critical path, $C P$, a convention we adopt hereinafter.

Our problem is to analyse the effects of choices of overtime assignments, each of which seeks to minimize project duration, risk of overrun and the amount of overtime deployed. This can be formulated as a three objective decision problem in which the three objectives of duration, risk and overtime are conflicting minimisation objectives.

We represent a candidate solution to our problem as an assignment of overtime to work packages. A feasible solution is an assignment of a certain number of extra hours to each work package, denoted by Overtime $\left(w p_{i}\right)$ subject to the following constraint: $0 \leq$ Overtime $\left(w p_{i}\right) \leq \operatorname{MaxOvertime}\left(w p_{i}\right)$, where MaxOvertime $\left(w p_{i}\right)$ is the maximum assignable overtime to the $i^{t h}$ work package and depends on the effort $e_{i}$ and the maximum overtime assignable per day ${ }^{1}$.

We shall use computational search to seek an allocation of overtime for all work packages that minimises each of the three objectives of Overtime $(O)$, Project Duration $(D)$ and Risk of Overrun $(R)$. We therefore measure fitness as a triple $\langle O, D, R\rangle$, whose components are defined as follows:

\footnotetext{
${ }^{1}$ The length of a working day and maximum allowed overtime are country specific parameters to our approach, determined by legal and governance procedures in place. In this paper we set these to 8 hours for a working day and 3 hours per day maximum overtime.
}

Overtime $(O)$ is the amount of time worked on each work package beyond the individual time limit per day summed over all work packages. More formally:

$$
O=\sum_{i=0}^{n} \text { Overtime }\left(w p_{i}\right)
$$

Project Duration $(D)$ is the estimated duration (i.e., the length of the critical path). More formally:

$$
D=\sum_{w p \in C P} \operatorname{Duration}(w p)
$$

We define the risk of overrun in terms of the risk of overrun associated to each path, $p$, in the project schedule:

$$
\text { risk }_{p}=\frac{\text { Duration }_{p}}{\text { Duration }_{C P}}
$$

The closer $r i s k_{p}$ is to 1.0 , the greater the chance that an overrun on a work package along path $p$ will cause $p$ to supersede the current critical path as the determinant of project duration ( $p$ thus becoming the new critical path due to the overrun).

We use three different approaches to the measurement of Risk of Overrun $(R)$, each of which combines the path risk $r i s k_{p}$, above into an overall project risk, $R$, as follows:

$$
\begin{array}{r}
R=R_{\text {AvgRisk }}=\frac{\sum_{p \in \Pi} \text { risk }_{p}}{|\Pi|} \\
R=R_{\text {MaxRisk }}=\max _{p \in \Pi-C P} \text { risk }_{p} \\
R=R_{\text {TrsRisk }}(L)=\frac{\mid\left\{p \cdot p \in \Pi \wedge \text { risk }_{p}>L\right\} \mid}{|\Pi|} \cdot 100
\end{array}
$$

These are, respectively, average, maximal, and threshold level risks. Average risk is suited to the engineer who is 'risk averse'; it assumes that any overrun on any path could be a problem. This is 'risk averse' in the sense that it reflects a pessimistic belief that 'anything that can go wrong will go wrong'. Maximum risk is better suited to the engineer who is more concerned that the critical path is not disrupted, but who is relaxed about overruns in non-critical paths that do not threaten to supersede the critical path, as these could be absorbed into the project schedule. Threshold risk allow the engineer to choose a risk level, making risk level a parameter to the overall approach (which we set to 0.75 in this paper).

Of course, overtime allocation is a disruptive process; it can change the critical path. This is one of the motivations for decision support: engineers cannot be expected to understand the impact of proposed overtime allocations on the critical path, while simultaneously balancing budgets, durations, and estimates of overruns. These are precisely those problems for which we need the kind of automated analysis we introduce in this paper.

\section{The SOlution APPROACH}

Our solution uses Search Based Software Engineering (SBSE) [16], [11], for which it is established best practice to define a representation, fitness function and computational search algorithm [17]. Since our formulation is a triple objective formulation we also need to decide how to handle multiple objectives.

Handling Multiple Objectives: In our case, the three objectives are measured on orthogonal scales so we use Pareto optimality, which states: "A solution $x_{1}$ is said to dominate another solution $x_{2}$, if $x_{1}$ is no worse than $x_{2}$ in all objectives and $x_{1}$ is strictly better than $x_{2}$ in at least one objective." 
Pareto optimality means that we do not suggest to the engineer a single proposed solution. That would not be realistic. No engineer would trust an automated tool to provide a single overtime allocation. Rather, we seek to provide a decision support tool, by showing the solutions in a space of trade offs between the three objectives, allowing the engineer to see the trade offs between them.

Using Pareto optimality we can plot the set of solutions found to be non-dominating (and therefore equally viable). In the case where there are three objectives, such as ours, this leads to a three dimensional Pareto surface, though we can also project this surface onto a two dimensional Pareto front to focus any two objectives of interest. The shapes of such surfaces and fronts can yield actionable insights. For example, where there is a knee point (a dramatic switch in the material values of trade off between objectives), this guides decision making (See Section V). Representation: Feasible solutions to the problem defined in Section II are assignments of a certain number of overtime hours to each work package. We encoded them as chromosomes of length $n$, where each gene represents the number of extra hours assigned to each work package. The initial population, composed by $n$ chromosomes, was randomly obtained by assigning to each $w p_{i}$ an overtime ranging from 0 to MaxOvertime $\left(w p_{i}\right)$. Fitness: To evaluate the fitness of each chromosome we employed a multi-objective function to simultaneously minimise the objectives described in Section II, namely Project Duration, Overtime, and Risk of Overrun. We report results for each overrun risk assessment measure (AvgRisk, MaxRisk, and TrsRisk) separately to explore the effects of each approach to risk assessment. Computational Search: As a ranking method, we employed a widely used Multi-Objective Evolutionary Algorithm (MOEA), namely NSGAII [18]. However, it is insufficient merely to apply a generic algorithm like NSGAII 'out of the box'; we need to define problem-specific genetic operators to ensure best performance. In the case of genetic algorithms such as NSGAII the crossover operator plays a pivotal role [19], [20], [21] and thus forms a natural focus for such problem-specific algorithm design.

We therefore introduce a variant of NSGAII (which we call $\mathrm{NSGAII}_{v}$ ) specifically for the overtime planning problem. $\mathrm{NSGAII}_{v}$ exhibits the same selection and crowding distance characteristics as the standard NSGAII but exploits a new crossover operator. Our crossover operator aims to preserve genes shared by the fittest overtime assignments, thereby avoiding the well-known disruptive effects of crossover [19]. It is defined as follows:

Let $P_{1}$ and $P_{2}$ be parent chromosomes, $C$ the point of cut randomly selected in the parents, and $O_{1}$ and $O_{2}$ the new offspring. For the genes placed before $C, O_{1}$ and $O_{2}$ inherit the genes of $P_{1}$ and $P_{2}$, respectively. While for each gene $g_{i}$ placed after $C$,

$$
\begin{gathered}
O_{1}\left(g_{i}\right)=\left\{\begin{array}{l}
\max \left(P_{1}\left(g_{i}\right), P_{2}\left(g_{i}\right)\right), p=0.5 \\
\min \left(P_{1}\left(g_{i}\right), P_{2}\left(g_{i}\right)\right), p=0.5
\end{array}\right\} \\
O_{2}\left(g_{i}\right)=\left(P_{1}\left(g_{i}\right)+P_{2}\left(g_{i}\right)\right) / 2
\end{gathered}
$$

Note that when the parent genes hold the same characteristic (i.e., same quantity of overtime) they are retained in both offspring, otherwise we generate two different genes for the offspring: one that inherits the gene from mother or father with equal probability and one that inherits both parent characteristics in terms of overtime average.

\section{The Design of THE EMPIRICAL STUdy}

This section explains the design of our empirical study; the research questions we set out to answer and the methods and statistical tests we used to answer these questions.

\section{A. Research Questions}

We seek to answer five research questions, each of which builds on its predecessor to develop the evidence for the validity, performance, usefulness, and insights gained from our approach to overtime planning.

RQ1 (SBSE Validation): How do NSGAII and $\mathrm{NSGAII}_{v}$ perform compared to random search? In any attempt at an SBSE formulation of a problem this is a standard 'baseline' question asked. If a proposed formulation does not allow an intelligent computational search technique to outperform random search convincingly, then there is clearly something wrong with the formulation. This question is thus adopted in SBSE research as a preliminary 'sanity check' [22].

RQ2.1 (Comparison to State of the Art Search): How does $\mathrm{NSGAII}_{v}$ perform compared to NSGAII? Outperforming random search is necessary, but not sufficient. In order for a proposed approach to be adopted it must also outperform the state of the art for the problem in hand. In this case, there is no prior work on the problem of planning overtime on software projects. We therefore compare our approach, $N S G A I I_{v}$, to the standard version of the algorithm (NSGAII), applied to our formulation. RQ2.2 (Usefulness): How does $\mathrm{NSGAII}_{v}$ perform compared to currently used overtime planning approaches? While outperforming a standard multi-objective search may be a valuable technical result, in order to be useful to software engineers, our approach must also outperform existing overtime management strategies used by practicing software engineers. We therefore repeat the experiments in RQ2.1, but for RQ2.2 we compare our approach with three currently used strategies. RQ3 (Insight): Can our approach yield useful insights into the trade offs between objectives for real world software projects? To provide decision support it is insufficient to demonstrate that our approach outperforms engineers' current practices. We must also provide evidence that our approach yields insights into the nature of the overtime planning problem. In order to do this, we need to give examples of actionable insights obtainable from the application of our approach to the real world software projects of the empirical study.

RQ4 (Impact of Risk Assessment Models): What is the difference between the three approaches to risk measurement? If our approach is demonstrated to outperform state of the art and current practice and it also provides useful insights, then we can use it to explore the three different risk assessment models we implemented (average, maximal, and threshold risk assessment). Since these different assessments of risk reflect different priorities in software engineers' management of risk, the findings shed light on the impact of different approaches to risk management in overtime planning.

\section{B. Software Projects Used in the Empirical Study}

Table I summarises the key information concerning the 6 projects used in the empirical study. The projects came from three different organisations, involved different kinds of software engineering development and had different sizes, ranging from 60 to 245 work packages and from a few person weeks to several person years in duration.

DB2 concerned the next release of a large data-intensive, multi-platform software system, written in several languages including DB II, SQL, and .NET. Web delivered a web-based IT sales system across North America. The project included the development and testing of website, search engine and order management and tracking. 
TABLE I

SOFTWARE PROJECTS USED IN THE EMPIRICAL STUDY. EFFORT IS MEASURED IN NORMALISED PERSON HOURS.

\begin{tabular}{|l||r|r|r|l|}
\hline Project & \#WPs & \#Dependencies & \multicolumn{1}{|c|}{ Effort } & Brief Description. \\
\hline \hline DB2 & 120 & 102 & 594 & A multi-platform database upgrade involving several languages such as DB2, SQL and .NET \\
\hline Web & 245 & 247 & 6,664 & A web-based purchase order system development \\
\hline Quote & 60 & 64 & 547 & An enhancement of an existing system to include on-demand conversion of quotes to orders \\
\hline Oracle & 106 & 105 & 5,390 & A large-scale Oracle database migration with tight data security constraints \\
\hline Price & 72 & 71 & 1,570 & A client-side sales system upgrade to offer additional features to users \\
\hline CutOver & 95 & 68 & 2,356 & Details cannot be revealed because of a Non Disclosure Agreement with the project data provider \\
\hline
\end{tabular}

Quote was a system developed for a large Canadian sales company to provide on-demand conversion of quotes to orders. Oracle was large scale database upgrade, migrating an old, yet mission-critical, Oracle system. The information that was migrated had an estimated value to the organisation of several million dollars and formed the cornerstone of the organisation's operations. Price was an enhancement to the client side of a sales system to provide improved pricing and features for discounts, vouchers, and price conversions. The details of project CutOver are the subject of a Non-Disclosure agreement and so cannot be published.

\section{Multi-objective Evaluation Measurements Used}

Assessing the performance of a computational search algorithm for a single objective optimisation problem typically requires observations about the best solution found. This approach is not applicable for multi-objective optimisation problems because there are a set of candidate solutions, each of which is said to be 'non-dominating'. That is, each is incomparable to the others because no other solution has better values for all objectives.

Analysis of graphical plots of the solutions can provide some indications of performance, but it provides a qualitative evaluation and cannot provide a quantitative assessment of the quality of solutions from one approach relative to another. A robust evaluation requires that qualitative evaluations be augmented by a more quantitive evaluation.

To provide this quantitative assessment we employ three solution set quality indicators, namely Contributions $\left(I_{C}\right)$, Hypervolume $\left(I_{H V}\right)$, and Generational Distance $\left(I_{G D}\right)$. To compute these we normalise fitness values to avoid unwanted scaling effects [23] and compute a reference front of solutions, $R S$, which is the set of non-dominated solutions found by the union of all approaches compared [24].

The $I_{C}$ quality indicator is the simplest measure. It measures the proportion of solutions given by an algorithm, $A$, that lie on the reference front $R S$ [25]. The higher this proportion, the more $A$ contributes to the best solutions found by the approaches compared, and so the better is the quality of its solutions. $I_{C}$ is a simple and intuitive measure, but it is affected by the number of solutions produced, unfavourably penalising algorithms that might produce 'few but excellent' solutions. This is why we also consider two other measures of solution quality, $I_{H V}$ and $I_{G D}$.

The $I_{H V}$ quality indicator [26] calculates the volume (in the objective space) covered by members of a non-dominated set of solutions from an algorithm of interest. The larger this volume, the better the algorithm, because the more it captures of the non-dominated solution space. Zitzler demonstrates [27] that this hypervolume measure is also strictly 'Pareto compliant'. That is, the hypervolume of $A$ is higher than $B$ if the Pareto set of $A$ dominates that of $B$. By using a volume rather than a count, this measure is also less susceptible to bias when the numbers of points on the two compared fronts are very different.
The $I_{G D}$ quality indicator [28] computes the average distance between the set of solutions, $S$, from the algorithm measured and the reference set $R S$. The distance between $S$ and $R S$ in an $n$ objective space is computed as the average $n$-dimensional Euclidean distance between each point in $S$ and its nearest neighbouring point in $R S$. We can think of $I_{G D}$ as the distance between the front $S$ and the reference front $R S$ in the $n$-dimensional objective space of the problem.

\section{Inferential Statistical Test Methods Used}

Due to the stochastic nature of evolutionary algorithms, best practice requires the use of careful deployment of inferential statistical testing to assess the differences in the performance of the algorithms used [17], [29]. We therefore performed 30 independent runs per algorithm, per risk assessment measure, per project to allow for such statistical testing.

To analyse the normality of distributions we employed the Shapiro test [30]. As we expected, many of our samples showed no evidence that they come from normally distributed populations, making the $t$-test unsuitable. We therefore used the Wilcoxon test [31] to check for statistical significance. Using the Wilcoxon test is a safe test to apply (even for normally distributed data), since it raises the bar for significance, by making no assumptions about underlying data distributions. We set the confidence limit, $\alpha$, at 0.05 and applied the standard Bonferroni correction $(\alpha / K$, where $K$ is the number of hypothesis) in cases where multiple hypotheses were tested.

As has been previously noted in advice on statistical testing of algorithms such as these [29], it is inadequate to merely show statistical significance alone; we also need to know whether the effect size is worthy of interest. Therefore, in order to additionally assess the effect size, we used Cohen's $d$, the results of which are usually considered small for $0.2 \leq d<0.5$, medium for $0.5 \leq d<0.8$, and large for $d \geq 0.8$ [31].

To answer RQ1 we implemented a random search to be compared with NSGAII and $\mathrm{NSGAII}_{v}$. The random search assigns randomly to each work package of the project, an overtime varying from 0 to the maximum overtime assignable. The resulting Pareto fronts were compared for statistically significant differences with those produced by NSGAII and $\mathrm{NSGAII}_{v}$, using the quality indicators explained in Section IV-C. In this sanity check we used the Wilcoxon test and for significance also performed a Cohen effect size test in all results.

We do not wish to devote too much space to RQ1, since it is only a 'sanity check', preferring to devote more space to the answers to RQs $2-4$, which concern more scientifically important evidence for the performance and usefulness of our approach. To answer RQ2.1 we compared NSGAII and $\mathrm{NSGAII}_{v}$ in terms of the quality indicators for statistical significance and effect size, as for RQ1, but additionally presenting the results using boxplots to give a pictorial account of the distributions of results obtained. 
TABLE II

CONFIGURATIONS EXPLORED TO TUNE THE TWO ALGORITHMS

\begin{tabular}{|l||r|r|r|}
\hline Configuration & Pop. Size & Generations & Fitness Evals \\
\hline \hline Very Small (VS) & 50 & 5,000 & 250,000 \\
\hline Small (S) & 100 & 2,500 & 250,000 \\
\hline Medium (M) & 200 & 1,250 & 250,000 \\
\hline Large (L) & 500 & 500 & 250,000 \\
\hline Very Large (VL) & 1,000 & 250 & 250,000 \\
\hline
\end{tabular}

TABLE III

BEST OBTAINED CONFIGURATIONS FOR NSGAII AND NSGAII

\begin{tabular}{|l||c|c|c|c|c|c|}
\hline \multirow{2}{*}{ Project } & \multicolumn{2}{c|}{ MaxRisk } & \multicolumn{2}{c|}{ AvgRisk } & \multicolumn{2}{c|}{ TrRRisk } \\
& NSGAII & NSGAII $_{v}$ & NSGAII & NSGAII & NSGAII & NSGAII $_{v}$ \\
\hline \hline DB2 & M & VL & VL & VL & L & VL \\
\hline Web & S & S & VL & VL & M & M \\
\hline Quote & VL & VL & VL & VL & VL & VL \\
\hline Oracle & VL & VL & VL & VL & S & S \\
\hline Price & L & VL & VL & VL & M & M \\
\hline CutOver & S & VS & VS & VL & VL & S \\
\hline
\end{tabular}

To answer RQ2.2 we compared NSGAII $v$ to standard overtime management strategies. That is, we implemented three strategies currently used, and compared the results to our $\mathrm{NSGAII}_{v}$ approach using the same tests as we performed to answer RQ2.1.

To answer RQ3 we analysed the Pareto fronts produced by our approach in order to identify useful trade offs among different goals and to discover knee points by means of graphical plots.

We answer RQ4 by exploring the differences between the three risk assessment approaches studied in the paper (in terms of the Pareto surfaces that capture the solution space for each form of assessment).

\section{E. Parameter Tuning and Setting}

An often overlooked aspect of research on computational search algorithms lies in the selection and tuning of the algorithmic parameters, which is necessary in order to ensure fair comparison, but which often goes unreported and, thereby, hinders any potential replication. In order to facilitate replication of our findings, we report the method adopted for algorithmic parameter tuning and selection in this section.

We evaluated, for each algorithm, five different configurations characterised by very small, small, medium, large, and very large values for population as detailed in Table II. All configurations were allowed an identical budget of fitness evaluations $(250,000)$, thereby ensuring that all require the same computational effort, though they may differ in parameter settings. We executed NSGAII (NSGAII $v$ ) with each configuration 30 times and collected the corresponding $I_{C}, I_{H V}$, and $I_{G D}$ values, testing for significant differences using the Wilcoxon Test. Table III reports the best configurations we selected for each algorithm and risk measure.

The rest of our parameter settings for both algorithms were typical standard settings. We report them here for completeness and replicability. For population size $n$, at each generation, $n / 2$ applications of the single point crossover operator are used to construct offspring. The crossover operator performs crossover with a probability 0.5 . The same number of mutations were performed, where the value of each gene is modified with a probability of 0.3 . The mutation operator randomly assigns a new value between 0 and MaxOvertime $(w p)$. We employed binary tournament selection based on dominance and crowding distance, and in tied tournaments one of the two competitor parents is chosen at random (with equal probability for both).

\section{AnAlysis of Results}

This section presents the results obtained from our experiments for RQs 1-4 set out in Section IV-A.
TABLE IV

THE SIGNIFICANTLY BETTER ALGORITHM (EFFECT SIZE IN PARENTHESES)

\begin{tabular}{|c|c|c|c|c|}
\hline Project & Risk Measure & $I_{C}$ & $I_{H V}$ & $I_{G D}$ \\
\hline \multirow{3}{*}{ DB2 } & AvgRisk & No sign. diff. (small) & NSGAII $_{v}$ (small) & NSGAII $_{v}$ (small) \\
\hline & MaxRisk & NSGAII $_{v}$ (high) & NSGAII $_{v}$ (high) & No sign. diff. (small) \\
\hline & TrsRisk & NSGAII $_{v}$ (high) & NSGAII $_{v}$ (small) & No sign. diff. (small) \\
\hline \multirow[t]{3}{*}{ Web } & AvgRisk & NSGAII $_{v}$ (high) & NSGAII $_{v}$ (high) & NSGAII $_{v}$ (high) \\
\hline & MaxRisk & NSGAII $_{v}$ (high) & NSGAII $_{v}$ (high) & NSGAII $_{v}$ (high) \\
\hline & TrsRisk & NSGAII $_{v}$ (high) & NSGAII $_{v}$ (high) & NSGAII $_{v}$ (high) \\
\hline \multirow[t]{3}{*}{ Quote } & AvgRisk & $\operatorname{NSGAII}_{v}$ (high) & $\operatorname{NSGAII}_{v}$ (high) & NSGAII $_{v}$ (high) \\
\hline & MaxRisk & No sign. diff. (small) & $\operatorname{NSGAII}_{v}$ (small) & No sign. diff. (small) \\
\hline & TrsRisk & $=$ & $=$ & $=$ \\
\hline \multirow[t]{3}{*}{ Oracle } & AvgRisk & No sign. diff. (small) & NSGAII $_{v}$ (small) & No sign. diff. (small) \\
\hline & MaxRisk & NSGAII $_{v}$ (high) & NSGAII $_{v}$ (high) & NSGAII $_{v}$ (high) \\
\hline & TrsRisk & $=$ & $=$ & $=$ \\
\hline \multirow[t]{3}{*}{ Price } & AvgRisk & NSGAII $_{v}$ (high) & NSGAII $_{v}$ (high) & NSGAII $_{v}$ (high) \\
\hline & MaxRisk & NSGAII $_{v}$ (high) & NSGAII $_{v}$ (high) & NSGAII $_{v}$ (high) \\
\hline & TrsRisk & NSGAII $_{v}$ (high) & NSGAII $_{v}$ (high) & NSGAII $_{v}$ (high) \\
\hline \multirow[t]{3}{*}{ CutOver } & AvgRisk & NSGAII $_{v}$ (high) & NSGAII $v$ (high) & NSGAII $_{v}$ (high) \\
\hline & MaxRisk & NSGAII $_{v}$ (high) & NSGAII $_{v}$ (high) & NSGAII $_{v}$ (high) \\
\hline & TrsRisk & $\operatorname{NSGAII}_{v}$ (high) & $\operatorname{NSGAII}_{v}$ (high) & NSGAII $_{v}$ (high) \\
\hline
\end{tabular}

Results for RQ1 (SBSE Validation): We observed that both NSGAII and NSGAII $v$ achieved superior values compared to random search for all three quality indicators (i.e., $I_{C}, I_{H V}$, $\left.I_{G D}\right)$ and on all six projects. The Wilcoxon test results showed that for all 54 experiments ( 3 indicators, 3 risk assessment approaches, and 6 projects) the quality indicators achieved by NSGAII and NSGAII $v$ were significantly better than those of random search with a Cohen effect size 'high'. Thus, we conclude that there is strong empirical evidence that our formulation passes the sanity check denoted by RQ1.

Results for RQ2.1 (Comparison to State of the Art Search): Table IV presents the results of the significance tests and effect size test. We observe that our algorithm, $\mathrm{NSGAII}_{v}$, outperforms the standard NSGAII in 41 out of $54(76 \%)$ experiments and in 35 of these 41 (85\%) it does so with a Cohen effect size 'high', whilst the standard approach does not outperform our approach in any of the experiments.

In more detail, we observe that $\mathrm{NSGAII}_{v}$ achieved significantly superior $I_{C}$ values with respect to NSGAII in 13 out 18 cases with a high effect size. Only in two cases (i.e., projects Quote and Oracle with TrsRisk) the two distributions were identical, while in three cases (i.e., projects DB2, Quote, and Oracle with AvgRisk, MaxRisk, and AvgRisk, respectively) no significant differences were found. For $I_{H V}$, we observe that NSGAII $v$ significantly outperformed NSGAII in 16 out of 18 cases with a high and small effect size (12 and 4 respectively), while in the remaining two cases (i.e., projects Quote and Price with TrsRisk) the two distributions were identical. For the third quality evaluation measurement, $I_{G D}$, $\mathrm{NSGAII}_{v}$ achieved significantly better values than NSGAII in 11 out of 18 cases with a high effect size and in one further case there was a significant difference (but small effect size). In 4 cases there were no significant differences between the two approaches and, again, on projects Quote and Oracle with TrsRisk the two distributions were identical.

We can also get a more qualitative sense of the distributions of results for the two approaches from the box plots shown in Figures 1, 2 and 3. From these box plots we can see that the variance in the results from both algorithms is lower for the threshold risk assessment measure than the other two. Subsequent results for RQ3 and 4 (see below) suggest that this is because there are simply fewer solutions available when threshold risk is used, due to the constraints the threshold imposes. For the other two risk assessment measures, variance is project specific, but low in all cases irrespective of the project concerned: standard deviation values ranged between 0.01 to 0.06 overall projects except DB2 and Quote (for which it was always lower than 0.01). 


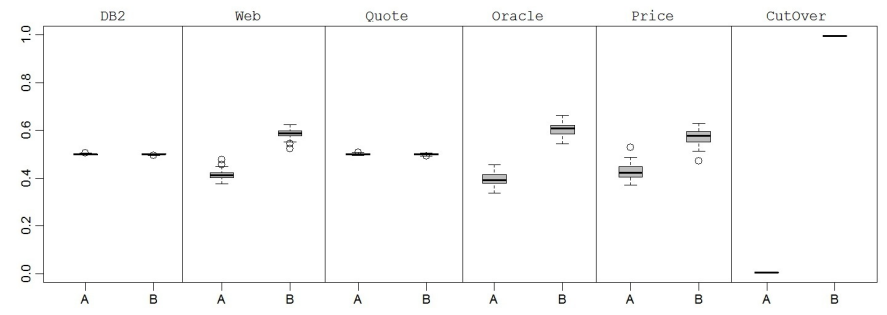

(a)

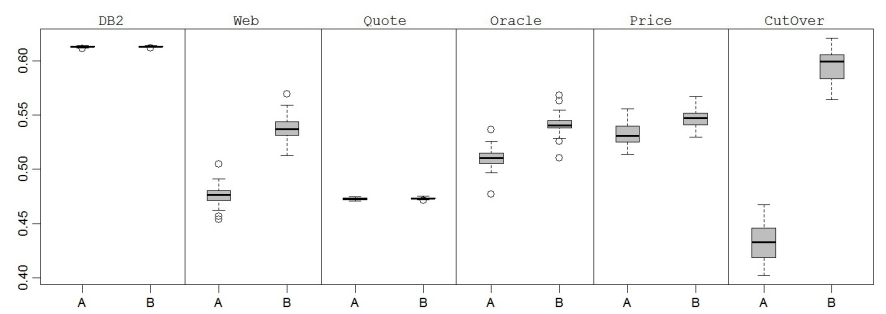

(b)

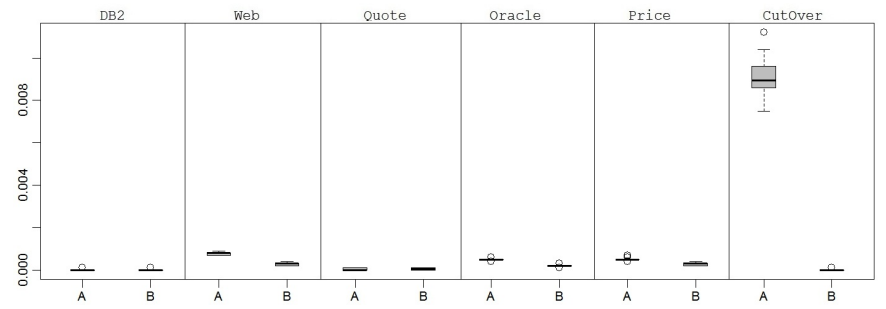

(c)

Fig. 1. Boxplots for the average risk assessment approach (AvgRisk), evaluated using the quality measures $I_{C}(\mathrm{a}), I_{H V}(\mathrm{~b})$, and $I_{G D}(\mathrm{c})$ applied to NSGAII (Algorithm A) and NSGAII $v$ (Algorithm B)

Results for RQ2.2 (Usefulness): In order to answer RQ2.2, we need to define 'current overtime planning practice'. There is evidence that current overtime practice employs what we term 'margarine management'; spreading the overtime thinly and evenly over all work packages [32]. We can therefore compare our approach to this documented Overtime Management Strategy (OMS). There are two other natural strategies (often referred to anecdotally in the literature): loading overtime onto the critical path to reduce completion time and loading it onto the later half of the project to compensate for earlier delays.

Table $\mathrm{V}$ reports the mean values of each of the three quality assessment indicators obtained for 30 runs of all projects using $\mathrm{NSGAII}_{v}$ and the three OMS practices defined above. The Wilcoxon Test confirmed that all the indicators obtained employing $\mathrm{NSGAII}_{v}$ were significantly better than those obtained with each and all of the OMS practices and with a high Cohen effect size in every case.

As an example, Figure 4 shows the reference fronts obtained by $\mathrm{NSGAII}_{v}$ and the three OMS practices for the largest project Web. In this and all subsequent figures, overtime is measured in total overtime hours committed to the project, while project duration is measured as the length of the critical path (in days).

While Table V gives the precise technical answer to RQ2.2, Figure 4 provides a more qualitative assessment of the meaning of this technical finding. As can be seen, the Pareto surface produced by $\mathrm{NSGAII}_{v}$ offers many more points (sometimes revealing quite clearly the shape of the solution space).

By contrast, the currently used approaches appear to merely

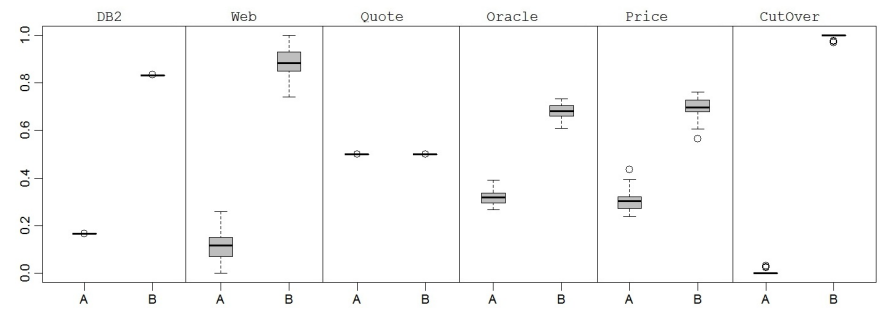

(a)

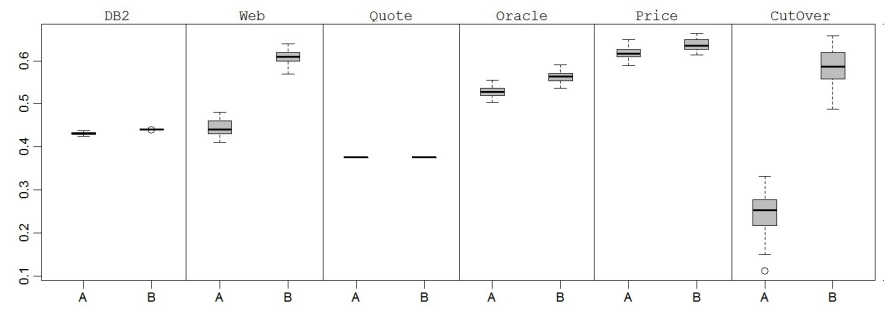

(b)

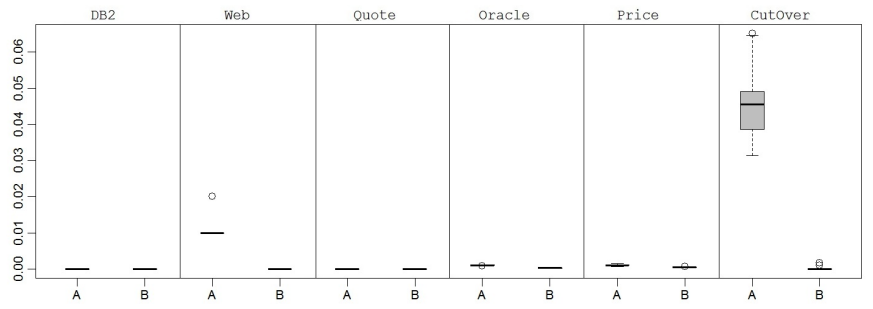

(c)

Fig. 2. Boxplots for the maximal risk assessment approach (MaxRisk), evaluated using the quality measures $I_{C}(\mathrm{a}), I_{H V}(\mathrm{~b})$, and $I_{G D}(\mathrm{c})$ applied to NSGAII (Algorithm A) and NSGAII $v$ (Algorithm B)

TABLE V

MEAN VALUES OF THE QUALITY INDICATORS FOR NSGAII $v$ AND THE THREE CURRENT OVERTIME MANAGEMENT STRATEGIES (OMS).

\begin{tabular}{|c|c|c|c|c|c|c|c|}
\hline Project & \multirow{2}{*}{ Risk Measure } & \multicolumn{2}{|c|}{$I_{C}$} & \multicolumn{2}{c|}{$I_{H V}$} & \multicolumn{2}{c|}{$I_{G D}$} \\
& & NSGAII $_{v}$ & OMS & NSGAII $_{v}$ & OMS & NSGAII $_{v}$ & OMS \\
\hline DB2 & AvgRisk & 0.998 & 0.002 & 0.614 & 0.259 & 0.001 & 0.070 \\
\hline & MaxRisk & 0.998 & 0.002 & 0.440 & 0.116 & 0.000 & 0.237 \\
\hline & TrsRisk & 0.999 & 0.001 & 0.461 & 0.000 & 0.000 & 0.456 \\
\hline Web & AvgRisk & 0.993 & 0.007 & 0.544 & 0.226 & 0.000 & 0.016 \\
\hline & MaxRisk & 0.962 & 0.038 & 0.559 & 0.257 & 0.008 & 0.195 \\
\hline & TrsRisk & 0.961 & 0.039 & 0.551 & 0.203 & 0.001 & 0.060 \\
\hline Quote & AvgRisk & 0.997 & 0.003 & 0.475 & 0.269 & 0.000 & 0.013 \\
\hline & MaxRisk & 0.997 & 0.003 & 0.376 & 0.148 & 0.000 & 0.128 \\
\hline & TrsRisk & 0.998 & 0.002 & 0.424 & 0.116 & 0.000 & 0.161 \\
\hline Oracle & AvgRisk & 0.994 & 0.006 & 0.611 & 0.329 & 0.000 & 0.013 \\
\hline & MaxRisk & 0.994 & 0.006 & 0.630 & 0.256 & 0.000 & 0.033 \\
\hline & TrsRisk & 0.916 & 0.084 & 0.645 & 0.371 & 0.007 & 0.038 \\
\hline Price & AvgRisk & 0.994 & 0.006 & 0.583 & 0.247 & 0.000 & 0.024 \\
\hline & MaxRisk & 0.992 & 0.008 & 0.696 & 0.428 & 0.000 & 0.032 \\
\hline & TrsRisk & 0.987 & 0.013 & 0.346 & 0.004 & 0.001 & 0.116 \\
\hline CutOver & AvgRisk & 0.996 & 0.004 & 0.609 & 0.305 & 0.000 & 0.028 \\
\hline & MaxRisk & 0.910 & 0.090 & 0.544 & 0.260 & 0.015 & 1.285 \\
\hline & TrsRisk & 0.970 & 0.030 & 0.700 & 0.277 & 0.002 & 0.235 \\
\hline
\end{tabular}

pick relatively arbitrary solutions, which are sub-optimal (far away from the frontier) and which thus denote little more than rather inaccurate guesses.

Results for RQ3 (Insight): To answer RQ3 we present examples of the Pareto fronts obtained using $\mathrm{NSGAII}_{v}$, illustrating how they reveal insights into the trade off between risk, duration, and overtime. Software engineers can exploit this information when making decisions about software project overtime planning. For example, Figure 5 reports the two-dimensional projections of the Pareto front obtained by executing $\mathrm{NSGAII}_{v}$ on the Price project, projecting the result onto the two objectives of overtime and risk. Such a 2D projection depicts the tradeoff between the spend on the overtime 


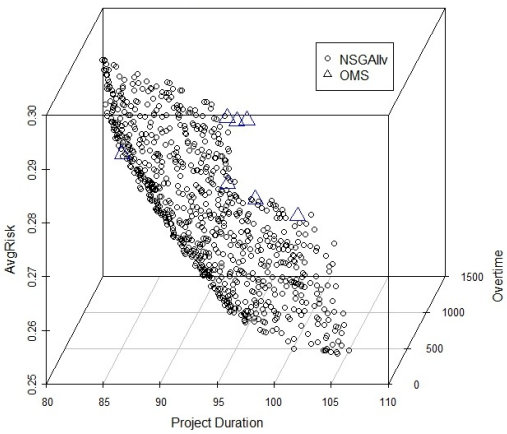

(a)

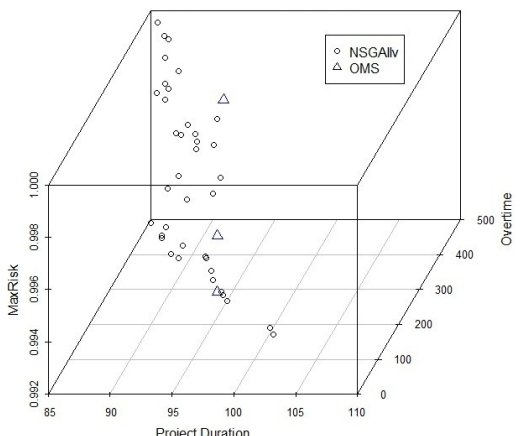

(b)

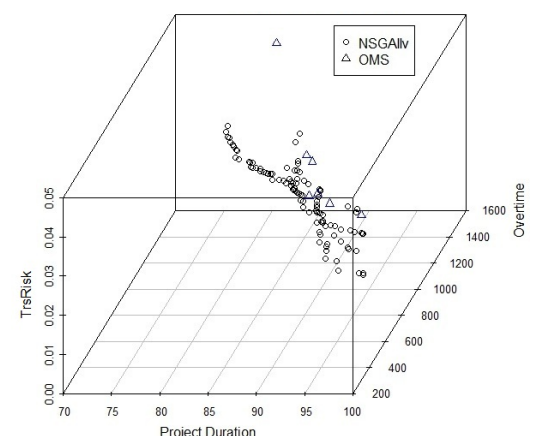

(c)

Fig. 4. Pareto surfaces for $\mathrm{NSGAII}_{v}$ (depicted by the circles) and for all of the three Overtime Management Strategies (depicted by the triangles) obtained using each of the three risk assessment approaches: AvgRisk(a), MaxRisk(b), and TrsRisk(c) for the project Web.

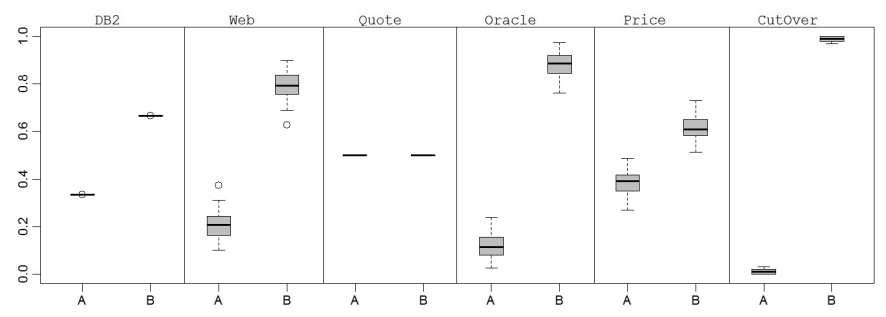

(a)

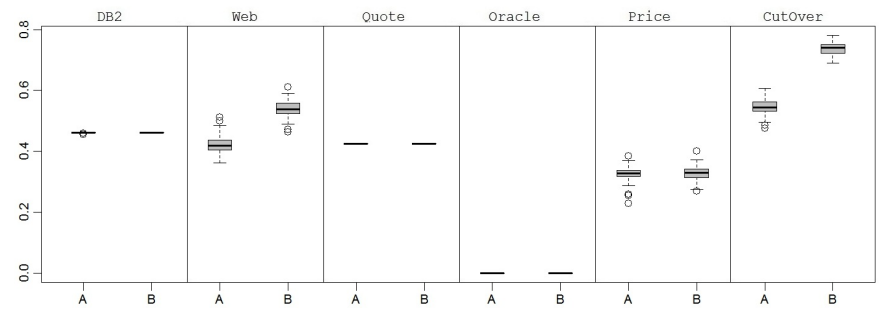

(b)

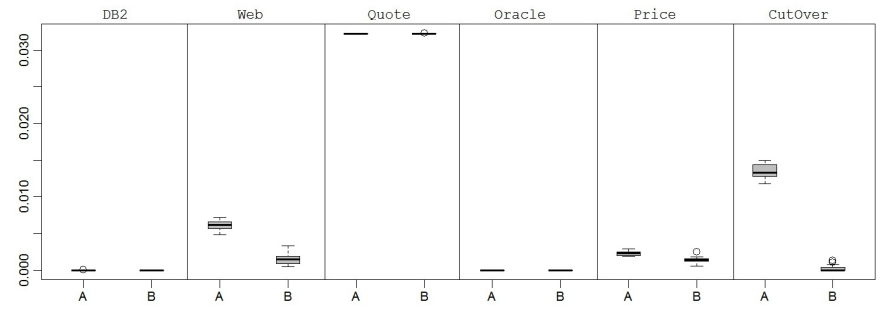

(c)

Fig. 3. Boxplots for the threshold risk assessment approach (TrsRisk), evaluated using the quality measures $I_{C}(\mathrm{a}), I_{H V}(\mathrm{~b})$, and $I_{G D}(\mathrm{c})$ applied to NSGAII (Algorithm A) and NSGAII $v$ (Algorithm B)

budget and the impact this has on reducing risk according to the three risk assessment measures. Several insights are immediately obvious from these three plots. We can see that using a threshold risk level reduces the scope for choice in selection of an overtime budget (fewer points on the Pareto front in Figure 5(c)). Such a threshold should thus only be considered by an engineer who is certain they have selected an appropriate risk level.

By contrast, the risk averse engineer who seeks to reduce average risk overall, is presented with a relatively smooth trade off between the cost of overtime and the risk reduction it affords (Figure 5(a)).

For the engineer who seeks to reduce only the maximal risk there is clear guidance from the shape of the Pareto front for Figure 5(b): A knee point in the front occurs at a budget of around 25 hours overtime. Up to this knee point along the horizontal axis, modest increases in overtime spend will yield sharp corresponding reductions in maximal risk. However, increasing overtime spend above this knee point of 25 hours, the engineer has to commit significantly more overtime spend to further reduce risk.

This finding has actionable conclusions for the software engineer planning overtime on this project. Without the benefit of the insights provided by our overtime planning analysis, it would naturally be tempting to seek the maximum overtime budget allowable (202 hours) to ensure that there is the largest resource available to deal with problems. However, this inherently conservative approach will likely lead to stress, illness and defects as widely reported in the literature [1], [2], [3], [4], [6], [7], [8].

Fortunately, with the benefit of our overtime planning analysis, it is revealed that there is a much more compelling alternative. With a budget of only 25 hours overtime (only about $12 \%$ of the maximum allowable) it is possible to achieve more than $50 \%$ of the risk reduction achievable by the conservative approach which maximises overtime to minimise overrun risk. Of course, it will be for the software engineer to make the final decision concerning overtime planning. However, using our analysis, he or she can clearly see that a budget of around $12 \%$ of the maximum overtime allowable would be ideal in principle and this is an important input to the final decision, bearing in mind the well-known problems associated with overuse of overtime.

Using these Pareto fronts the software engineer can thus obtain answers to questions like 'how much spend on overtime is cost effective for my project plan?' and 'what must I spend to reduce average risk by $x \%$ ?'. Such answers provide decision support to the engineer faced with the task of negotiating an overtime budget with their manager. They may also support decisions about how to most effectively deploy a budget to reduce risk. Space does not permit us to show results for all 6 projects. However, similar examples of interesting insights were observed for other projects. Results for RQ4 (Impact of Risk Assessment Models): To answer RQ4 we use the 3D visualisations of the Pareto surface, thereby showing the trade offs between all three objectives of overtime, duration, and risk. This allows us to compare the influence of the three different risk assessment measurements. Once again, space does not allow us to present all 18 plots, so we present (in Figure 6) the results from one project (Price) that is typical of the overall trends we observed in all projects.

The most immediately obvious effect is the number of 


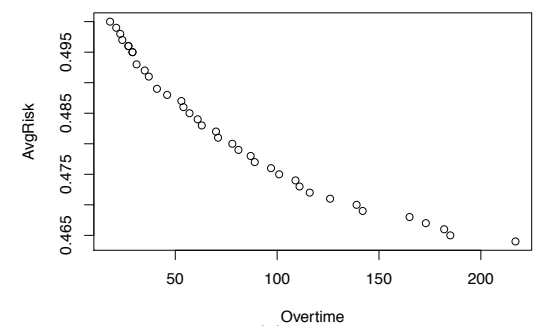

(a)

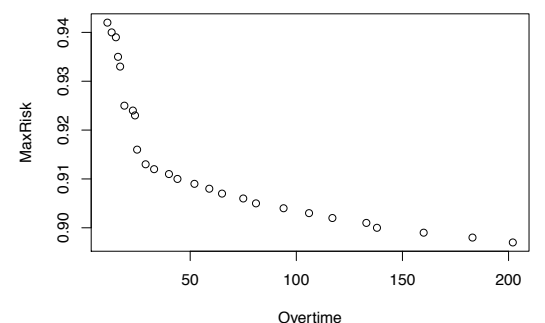

(b)

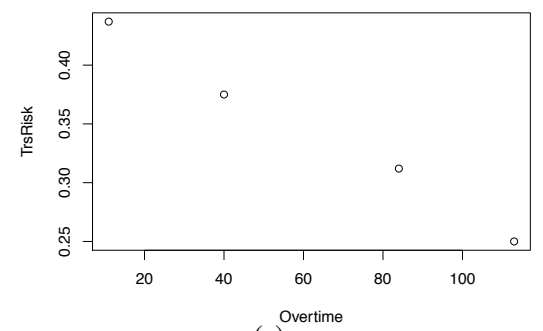

(c)

Fig. 5. 2D Pareto surface projections for each of the three risk assessment approaches: AvgRisk(a), MaxRisk(b) and TrsRisk(c) for the project Price.

solutions offered by each approach to risk assessment. This can be clearly observed from the density of the Pareto surfaces shown in Figure 6 and it reveals an inherent property of these three risk assessment methods: the AvgRisk surface has 2,452 solutions, while MaxRisk has 1,228 and TrsRisk has only 182 . Over all six projects the median number of solutions found was 2,535 for AvgRisk, 1,473 for MaxRisk and 190 for TsrRisk indicating that Figure 6 is not atypical.

The risk averse planner seeks to reduce average risk over all project paths and so this leads to many different trade offs and the largest number of potential solutions. The software engineer who has confidence in the applicability of the Critical Path Method (and thus seeks to reduce risks along this path) has fewer solutions available. However, this 'CPM-confident' engineer still has many solutions from which to choose and, more importantly, a continuous set of increasing degrees of trade off along each of the three dimensions of choice. By contrast, the engineer who is so confident in their risk assessment that they set a threshold risk level will have few solutions from which to choose; the threshold choice constrains the solutions. In particular, it reduces the number of projected project durations from which the engineer can choose.

The Pareto surfaces in Figure 6 also reveal an effect we found in four of the projects (Web, Oracle, Price and CutOver): the risk averse (AvgRisk) form of assessment leads to less pronounced changes in the dynamics of the trade off compared to the other two approaches. This can clearly be seen from the shape of Figure 6(a) compared (for example) to Figure 6(b); the former depicts a more gentle gradient through any arbitrary projection of the surface compared to the latter.

This finding reflects the fact that the risk averse engineer seeks to minimise all risks, thereby reducing difference between choices, whereas the engineer who focuses effort on the critical path has a more pronounced impact of the choices they make on the solutions offered. Of course, there is no 'free lunch': we can also see that the risk averse engineer typically has to commit greater resources to satisfy their strategy than the others (solutions appear further to the right, denoting high overtime costs, in Figure 6(a) compared to the other two). Therefore, we can see that risk aversion comes at an increased cost.

A Note on Project-Specific Effects: While examining the results to answer RQs2-4 we noted the effects due to the characteristics of the projects. In particular the results for DB2 and Quote appeared to differ from those of the other four. These two projects are much smaller than the others (594 person hours for DB2 and 547 for Quote compared to an average of 3,995 person hours for the other four projects). Their small size is reflected in the comparatively low variance of results for NSGAII and NSGA2 ${ }_{v}$ reported in answer to RQ2.1 (Figures 1, 2 and 3).

We also observed fewer differences in the three risk assessments reported in RQ4 and were able to find fewer examples of knee points and other insights obtainable for DB2 and Quote when answering RQ3. We therefore find that, unsurprisingly, smaller projects may not offer the software engineer as rich a set of overtime planning choices as large and more complex projects.

\section{Threats to VALIDITY}

It is widely recognised that several factors can bias the validity of empirical studies. In this section we discuss the validity of our study based on three types of threats, namely construct, internal, and external validity. Construct validity concerns the methodology employed to construct the experiment. Internal validity concerns possible bias in the way in which the results were obtained, while external validity concerns the possible bias of choice of experimental subjects.

In our study, construct validity threats may arise from the assumptions we make about the current state of the art and practice. Since we are the first to address this problem, there simply is no currently established state of the art in terms of computational search. We therefore compared our results for technical quality against a standard multi-objective approach, implemented and applied to the same subject set with the same settings. We also found comparatively little literature to guide us on what we should consider to be the 'standard practice' adopted by engineers. We followed the literature for one of these choices ('margarine management'), but there is only anecdotal evidence in the literature for the other two practices. By comparing our overtime planning approach to all three choices of OMS practice, we seek to compare with a set of alternative current practices for which there is some degree of support in the literature. Another threat to construct validity can arise from the fact that we did not take into account resource allocation and skills in the formulation of the problem.

We catered for internal threats to validity in the standard manner for randomised algorithms [29], [17], using non-parametric statistical testing over 30 repeated runs of the algorithms.

Our approach to external threats is also relatively standard for the empirical software engineering literature. That is, while we were able to obtain a set of subjects that had a degree of diversity in scope, application and project team, we cannot claim that our results generalise beyond these subjects studied.

\section{RELATED WORK}

For a long time, software engineers have used the Critical Path Method as the principle means of bringing some rudimentary analysis to bear on the problem of project planning [15]. Many software engineers use this approach to plan their projects. However, there have been attempts to replace the human project 


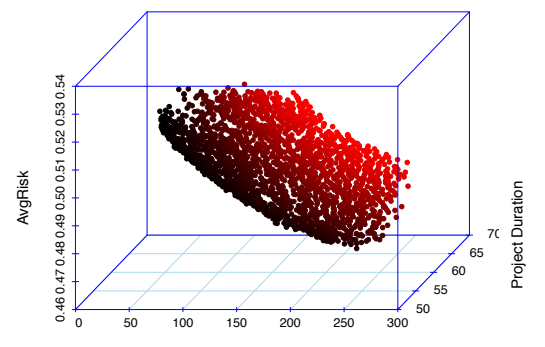

(a)

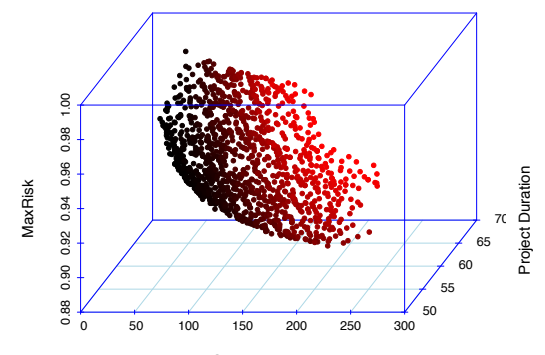

(b)

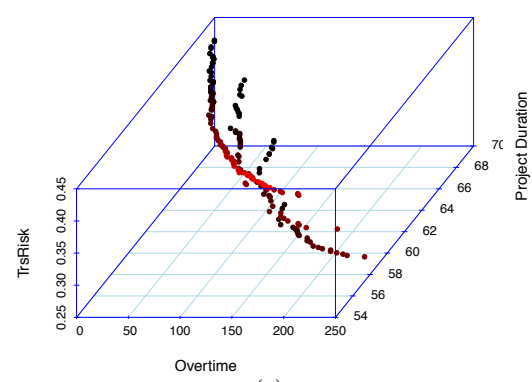

(c)

Fig. 6. Pareto surfaces for the project Price obtained using each of the three risk assessment approaches: AvgRisk(a), MaxRisk(b) and TrsRisk(c)

planner with a more automated planner, based on scheduling and resource optimisation techniques.

The first such attempt to apply optimisation to software project planning was the work of Chang et al. [33], who introduced the Software Project Management Net (SPMNet) approach for project scheduling and resource allocation and evaluated it on simulated project data. Subsequent research also formulated the problem of constructing an initial project plan as a Search Based Software Engineering (SBSE) problem, using scheduling and simulation [34], [35]. Though most approaches have focused on minimising project duration as the sole optimisation objective, there has also been work on constructing suitable teams of engineers [36], [37], [38].

Previous work has used a variety of SBSE techniques such as Genetic Algorithms [34], Simulated Annealing [36], Co-evolution [39] and Scatter Search [40] as well as hybrids, for example, combining SBSE with constraint satisfaction [41].

Though most of the previous work has been single objective, there has been previous work on multi-objective formulations [36], [42], [43]. However, unlike the present paper, none of this previous work has considered overtime, and all previous work starts with the assumption that it is the role of the optimisation tool (not the software engineer) to provide the initial project plan.

We believe that the assumption that any automated tool should have the role of producing the initial project plan, may not always be realistic. Our experience with practitioners is that they would prefer to trust in their own judgement for the initial project plan. This is because the allocation of staff to teams and teams to work packages involves all sorts of human and domain specific judgements for which an automated approach is ill-equipped and a human may be far more suitable.

By contrast, our approach to the overtime planning problem has a fundamentally different starting point and usage scenario in mind: We do not seek to replace the software engineer, nor to second guess their decisions. Rather, we seek to provide decision support in analysing the effects and trade offs in overtime planning. Few software engineers set out with the intention of coercing their team into unplanned overtime, but many well-intentioned and professional software engineers end up doing just that [1], [6]. We seek to provide decision support so that this can be properly planned and better informed by multi-objective risk analysis.

Other authors have considered overtime planning issues in software projects, though none has offered an approach to plan overtime, balancing overtime deployment against project risks. For example, Jia and Fan [44] analysed the use of System Dynamics Modeling [45], reporting results on a simulation carried out on a real software project (called ISAM3.1 at Alcatel
Shanghai Bell). They report on the harmful effects of excessive overtime (above set limits).

Lipke [46] presents a brief report of an effort to control the use of reserve budget in a software project for the defence industry. There are many authors who opine overtime's severe negative impacts on staff and their projects (e.g., [2], [3], [4]) but none offers a technique for automated decision support to help the engineer better plan the deployment of overtime.

We believe that ours is the first approach to address this issue, analysing overtime planning and its relationship to project duration and overrun risk. Furthermore, unlike previous work, because our approach starts with the software engineer's original project plan (rather than attempting to construct it), it requires no simulation, thereby removing this source of potential error and the assumptions that go with it.

\section{CONCLUSIONS AND FUTURE WORK}

We have introduced a search based approach to overtime planning on software engineering projects and evaluated it on 6 real world software engineering projects. Our approach performs significantly better (with high effect size) than currently used software engineering practice in terms of three standard measures of result quality. It also performs better than a standard multi-objective optimisation applied to the same problem for $76 \%$ of experiments (and never performs significantly worse).

We provide qualitative evidence that the approach can provide actionable insights to the software engineer, backing up this quantitative evidence that it is effective and useful. We also used our approach to explore the differences between risk assessment strategies revealing, inter alia, the increased costs that accrue from risk aversion and the paucity of choice available to managers who have precise risk thresholds in mind.

We believe that this paper lays a firm foundation for future development of semi-automated decision support for software engineers faced with the challenges of planning overtime on complex and demanding projects. However, there remains much to be done to realise the practical benefits that this approach offers.

In future work we plan to deploy a version of the tooling reported upon in this paper as a freely available, open source plug-in component to popular project planning tools, such as Microsoft project. This will allow more extensive evaluation of the interface between the technical aspects of the work reported in this paper and other related socio-technical issues for implementation and exploitation, such as user interface, HCI, and decision support. Moreover, this will allow us also to get feedback from practioners on the usefulness of the insights provided by our approach and the considered overrun risk strategies. We also 
plan to collect more data to analyze how well the model performs by applying it on actual projects and comparing the outcomes with projects that use the traditional rule-of-thumb strategies. Finally, it would be interesting to extend the problem formulation considering other aspects, such as resource allocation and skills.

Acknowledgments: The research is part funded by University of Salerno's Finanziamento Medie e Grandi Attrezzature 2010 and by EPSRC grants G060525 J017515.

\section{REFERENCES}

[1] E. Yourdon, Death March: The Complete Software Developer's Guide to Surviving 'Mission Impossible' Projects. Prentice-Hall, 1997.

[2] K. Driesen, N. W. H. Jansen, I. Kant, D. C. L. Mohren, and L. G. P. M. van Amelsvoort, "Depressed mood in the working population: associations with work schedules and working hours," Chronobiology International, vol. 27, no. 5, pp. 1062-1079, 2010.

[3] E. Kleppa, B. Sanne, and G. S. Tell, "Working overtime is associated with anxiety and depression: The Hordaland health study," Journal of Occupational and Environmental Medicine, vol. 50, no. 6, pp. 658-666, 2008.

[4] M. Van Der Hulst and S. Geurts, "Associations between overtime and psychological health in high and low reward jobs," Work and Stress: An International Journal of Work, Health and Organisations, vol. 15, no. 3, pp. 227-240, 2001.

[5] R. Pressman, Software Engineering: A Practitioner's Approach, 3rd ed Maidenhead, Berkshire, England, UK.: McGraw-Hill, 1992.

[6] M. Nishikitani, M. Nakao, K. Karita, K. Nomura, and E. Yano, "Influence of overtime work, sleep duration, and perceived job characteristics on the physical and mental status of software engineers," Industrial Health, vol. 43, no. 4, pp. 623-629, 2005.

[7] B. Akula and J. Cusick, "Impact of overtime and stress on software quality," in $4^{\text {th }}$ International Symposium on Management, Engineering, and Informatics (MEI 2008), Orlando, Florida, USA, 2008.

[8] C. Mann and F. Maurer, "A case study on the impact of scrum on overtime and customer satisfaction," in Agile Development Conference (ADC'05). Denver, Colorado, USA: IEEE Computer Society, 2005, pp. 70-79.

[9] D. G. Beckers, D. van der Linden, P. G. Smulders, M. A. Kompier, T. W. Taris, and S. A. Geurts, "Voluntary or involuntary? control over overtime and rewards for overtime in relation to fatigue and work satisfaction," Work and Stress: An International Journal of Work, Health and Organisations, vol. 22, no. 1, pp. 33-50, 2008.

[10] L. J. Osterweil, C. Ghezzi, J. Kramer, and A. L. Wolf, "Determining the impact of software engineering research on practice," IEEE Computer, vol. 41 , no. 3 , pp. $39-49,2008$.

[11] M. Harman, "The current state and future of search based software engineering," in Future of Software Engineering 2007, L. Briand and A. Wolf, Eds., 2007, pp. 342-357.

[12] G. Gay, T. Menzies, O. Jalali, G. Mundy, B. Gilkerson, M. S. Feather, and J. Kiper, "Finding robust solutions in requirements models," Automated Software Engineering, vol. 17, no. 1, pp. 87-116, March 2010.

[13] M. O. Saliu and G. Ruhe, "Bi-objective release planning for evolving software systems," in Proceedings of the $6^{\text {th }}$ joint meeting of the European Software Engineering Conference and the ACM SIGSOFT International Symposium on Foundations of Software Engineering (ESEC/FSE) 2007, I. Crnkovic and A. Bertolino, Eds. ACM, Sep. 2007, pp. 105-114.

[14] Y. Zhang, A. Finkelstein, and M. Harman, "Search based requirements optimisation: Existing work and challenges," in International Working Conference on Requirements Engineering: Foundation for Software Quality (REFSQ'08). LNCS, vol. 5025, 2008, pp. 88-94.

[15] J. E. Kelley, "The critical path method: Resource planning and scheduling," in Industrial Scheduling. Prentice Hall, 1963, pp. $347-365$.

[16] F. G. Freitas and J. T. Souza, "Ten years of search based software engineering: A bibliometric analysis," in $3^{\text {rd }}$ International Symposium on Search based Software Engineering (SSBSE 2011), 2011, pp. 18-32.

[17] M. Harman, P. McMinn, J. Souza, and S. Yoo, "Search based software engineering: Techniques, taxonomy, tutorial," in Empirical software engineering and verification: LASER 2009-2010, B. Meyer and M. Nordio, Eds. Springer, 2012, pp. 1-59, LNCS 7007.

[18] K. Deb, A. Pratap, S. Agarwal, and T. Meyarivan, "A fast and elitist multiobjective genetic algorithm: NSGA-II," IEEE Transactions on Evolutionary Computation, vol. 6, pp. 182-197, Apr. 2002.

[19] K. De Jong, "On using genetic algorithms to search program spaces," in Genetic Algorithms and their Applications: Proceedings of the second international Conference on Genetic Algorithms, J. J. Grefenstette, Ed. Hillsdale, NJ, USA: Lawrence Erlbaum Associates, 28-31 Jul. 1987, pp. 210-216.

[20] M. Harman and P. McMinn, "A theoretical and empirical study of search based testing: Local, global and hybrid search," IEEE Transactions on Software Engineering, vol. 36, no. 2, pp. 226-247, 2010.
[21] M. Harman, R. Hierons, and M. Proctor, "A new representation and crossover operator for search-based optimization of software modularization," in GECCO 2002: Proceedings of the Genetic and Evolutionary Computation Conference. San Francisco, CA 94104, USA: Morgan Kaufmann Publishers, 9-13 July 2002, pp. 1351-1358.

[22] M. Harman, A. Mansouri, and Y. Zhang, "Search based software engineering: Trends, techniques and applications," ACM Computing Surveys, 2012 , vol. 45 , no. 1 , p. 11.

[23] J. J. Durillo and A. J. Nebro, "jMetal: a Java framework for multi-objective optimization," Advances in Engineering Software, vol. 42, pp. 760-771, 2011.

[24] J. D. Knowles, L. Thiele, and E. Zitzler, "A tutorial on the performance assessment of stochastic multiobjective optimizers," Computer Engineering and Networks Laboratory, ETH Zurich, Tech. Rep. 214, 2006.

[25] H. Meunier, E.-G. Talbi, and P. Reininger, "A multiobjective genetic algorithm for radio network optimization," in Evolutionary Computation, 2000. Proceedings of the 2000 Congress on, vol. 1, 2000, pp. $317-324$

[26] E. Zitzler and L. Thiele, "Multiobjective evolutionary algorithms: a comparative case study and the strength pareto approach," IEEE Transactions on Evolutionary Computation, vol. 3, no. 4, pp. 257 -271, 1999.

[27] E. Zitzler, L. Thiele, M. Laumanns, C. Fonseca, and V. da Fonseca, "Performance assessment of multiobjective optimizers: an analysis and review," Evolutionary Computation, IEEE Transactions on, vol. 7, no. 2, pp. $117-132$, april 2003.

[28] D. A. V. Veldhuizen and G. B. Lamont, "Multiobjective evolutionary algorithm research: A history and analysis," 1998.

[29] A. Arcuri and L. Briand, "A practical guide for using statistical tests to assess randomized algorithms in software engineering," in $33^{\text {rd }}$ International Conference on Software Engineering (ICSE'11). ACM, 2011, pp. 1-10.

[30] P. Royston, "An extension of Shapiro and Wilk's W test for normality to large samples," Applied Statistics, vol. 31, no. 2, pp. 115-124, 1982.

[31] J. Cohen, Statistical power analysis for the behavioral sciences, 2nd ed. Lawrence Earlbaum Associates, 1988.

[32] A. S. Hanna and G. Haddadl, "Overtime and productivity in electrical construction," in Construction Research Congress, 2009, pp. 1-10.

[33] C. K. Chang, C. Chao, S.-Y. Hsieh, and Y. Alsalqan, "Spmnet: a formal methodology for software management," in $18^{t h}$ Annual International Computer Software and Applications Conference (COMPSAC '94). Taipei, Taiwan: IEEE, 9-11 November 1994, pp. 57-57.

[34] E. Alba and F. Chicano, "Software project management with gas," Information Sciences, vol. 177, no. 11, pp. 2380-2401, June 2007.

[35] G. Antoniol, M. D. Penta, and M. Harman, "Search-based techniques applied to optimization of project planning for a massive maintenance project," in $21^{\text {st }}$ IEEE International Conference on Software Maintenance, 2005, pp. 240-249.

[36] G. Antoniol, M. Di Penta, and M. Harman, "The use of search-based optimization techniques to schedule and staff software projects: An approach and an empirical study," Software - Practice and Experience, vol. 41, no. 5, pp. 495-519, April 2011.

[37] M. Hericko, A. Zivkovic, and I. Rozman, "An approach to optimizing software development team size," Information Processing Letters, vol. 108, no. 3, pp. 101-106, October 2008.

[38] D. Kang, J. Jung, and D.-H. Bae, "Constraint-based human resource allocation in software projects," Software: Practice and Experience, vol. 41, no. 5, pp. 551-577, April 2011.

[39] J. Ren, M. Harman, and M. Di Penta, "Cooperative co-evolutionary optimization on software project staff assignments and job scheduling," in $3^{\text {rd }}$ International Symposium on Search based Software Engineering (SSBSE 2011), 10th - 12th September 2011, pp. 127-141, LNCS Volume 6956

[40] R. Alvarez-Valds, E. Crespo, J. M. Tamarit, and F. Villa, "A scatter search algorithm for project scheduling under partially renewable resources," Journal of Heuristics, vol. 12, no. 1-2, pp. 95-113, March 2006.

[41] A. Barreto, M. de Oliveira Barros, and C. M. L. Werner, "Staffing a software project: a constraint satisfaction and optimization-based approach," Computers \& Operations Research, vol. 35, no. 10, pp. 3073-3089, October 2008

[42] F. Chicano, F. Luna, A. J. Nebro, and E. Alba, "Using multi-objective metaheuristics to solve the software project scheduling problem," in Proceedings of the 13th Annual Conference on Genetic and Evolutionary Computation (GECCO '11). Dublin, Ireland: ACM, 12-16 July 2011, pp. 1915-1922.

[43] G. Antoniol, S. Gueorguiev, and M. Harman, "Software project planning for robustness and completion time in the presence of uncertainty using multi objective search based software engineering," in ACM Genetic and Evolutionary Computation COnference (GECCO 2009), pp. 1673-1680.

[44] J. Jia, X. Fan, and Y. Lu, "System dynamics modeling for overtime management strategy of software project," in 25th International Conference of the System Dynamics Society, Boston, MA, USA, 2007, pp. 1-8.

[45] J. M. Lyneisa and D. N. Fordb, "System dynamics applied to project management: a survey, assessment, and directions for future research," System Dynamics Review, vol. 23, no. 2-3, pp. 157-189, 2007.

[46] W. H. Lipke, "Applying management reserve to software project management," Crosstalk: The Journal of Defense Software Engineering, pp. 17-21, 1999. 